\title{
MODIS and VIIRS On-orbit Calibration and Characterization Using Observations from Spacecraft Pitch Maneuvers
}

\author{
Xiaoxiong Xiong ${ }^{1}$, Aisheng $\mathrm{Wu}^{2}$, Amit Angal $^{2}$, Kwofu Chiang $^{2}$, and James Butler ${ }^{1}$ \\ ${ }^{1}$ Sciences and Exploration Directorate, NASA/GSFC, Greenbelt, MD 20771 \\ ${ }^{2}$ Science Systems and Applications Inc., 10210 Greenbelt Road, Lanham, MD 20706
}

\begin{abstract}
Two MODIS instruments (Terra and Aqua) and two VIIRS instruments (S-NPP and JPSS-1) are currently operated in space, continuously making global earth observations in the spectral range from visible (VIS) to long-wave infrared (LWIR). These observations have enabled a broad range of environmental data records to be generated and distributed in support of both operational and scientific community. Despite extensive pre-launch calibration and characterization performed for both MODIS and VIIRS instruments and routine on-orbit calibration activities carried out using their onboard calibrators $(\mathrm{OBC})$, various spacecraft maneuvers have also been designed and implemented to further enhance the sensor on-orbit calibration and data quality. This paper focuses on the use of observations made during spacecraft pitch maneuvers of MODIS and VIIRS in support of their on-orbit characterization of thermal emissive bands (TEB) response versus scan-angle (RVS). In the case of Terra MODIS, lunar observations made from instrument nadir view during spacecraft pitch maneuvers are used to compare with that made regularly through instrument space view (SV) port to evaluate on-orbit changes in RVS and band-to-band registration (BBR) for the reflective solar bands (RSB). In addition to results derived from spacecraft pitch maneuvers performed for MODIS and VIIRS, discussion is provided on the advantages, challenges, and lessons for future considerations and improvements.
\end{abstract}

Keywords: MODIS, VIIRS, maneuvers, lunar observations, calibration, RVS, BBR

\section{INTRODUCTION}

The Moderate Resolution Imaging Spectroradiometer (MODIS) is a key instrument for the NASA's Earth Observing System (EOS), currently operated on-board the Terra and Aqua spacecraft launched in December 1999 and May 2002, respectively (Figure 1) ${ }^{1,2}$. MODIS is a scanning radiometer using a two-sided scan mirror, collecting data in 36 spectral bands with wavelengths ranging from 0.41 to $14.5 \mu \mathrm{m}$ (Table 1). MODIS observations are made at 3 spatial resolutions (nadir): $250 \mathrm{~m}$ for bands 1-2 (40 detectors per band), $500 \mathrm{~m}$ for bands 3-7 (20 detectors per band), and $1 \mathrm{~km}$ for bands 836 (10 detectors per band). Bands 1-19 and 26 are the reflective solar bands (RSB) and bands 20-25 and 27-36 are the thermal emissive bands (TEB). Since launch, nearly 40 data products have been generated and distributed using both Terra and Aqua MODIS observations. These data products cover a wide range of applications and studies of the key properties or parameters of the Earth's land, ocean, and atmosphere ${ }^{3}$. 
The Visible/Infrared Imaging Radiometer Suite (VIIRS) instruments have been developed and designed with strong MODIS heritage by the same instrument vendor. It is also a scanning radiometer. Instead of a scan mirror, the VIIRS uses a rotating telescope assembly (RTA) coupled with a half-angle mirror (HAM). Currently, there are two operating VIIRS instruments, one on-board the Suomi National Polar-orbiting Partnership (S-NPP) spacecraft launched in October 2011 and another on-board the first Joint Polar Satellite System (JPSS) spacecraft launched in November 2017, now named as the NOAA-20 (N-20). VIIRS data are collected in 22 spectral bands covering wavelengths from 0.41 to 12.5 $\mu \mathrm{m}$ and at two spatial resolutions (nadir): $375 \mathrm{~m}$ for the imaging (I) bands and $750 \mathrm{~m}$ for the moderate (M) resolution bands. The VIIRS has a unique day/night band (DNB) that can make measurements over a very large dynamic range. Over 20 Environmental Data Records (EDR), with most similar to MODIS data products, can be generated from VIIRS observations, which support many applications by both operational and scientific research community ${ }^{4-6}$.
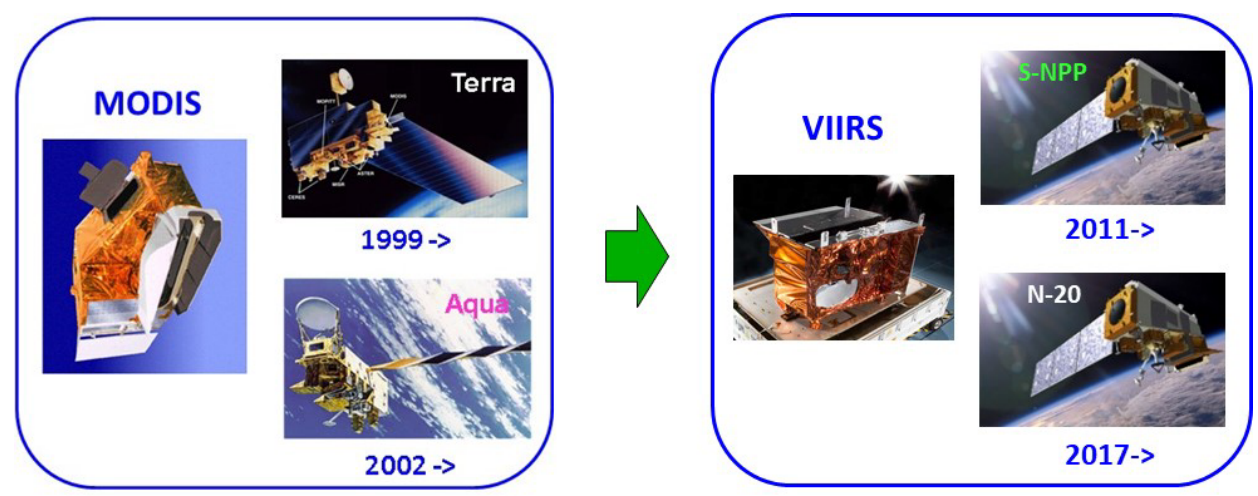

Figure 1 MODIS (left) on Terra and Aqua spacecraft launched in December 1999 and May 2002, respectively. VIIRS (right) on S-NPP and N-20 spacecraft launched October 2011 and November 2017, respectively.

Extensive pre-launch calibration and characterization efforts have been made for each MODIS and VIIRS instrument to evaluate its performance and to derive key sensor parameters that are critical for its on-orbit operation and calibration. In general, key sensor (or detector) performance parameters include but not limited to the following:

- Radiometric gain and nonlinearity

- Dynamic range

- Signal-to-noise ratio (SNR) and Nosie-equivalent temperature difference (NEdT)

- Relative spectral response (RSR)

- Response versus scan-angle (RVS)

- Band-to-band registration (BBR)

- Modulation transfer function (MTF)

- Polarization sensitivity

- Near field response (NFR)

To assure sensor data quality through the entire mission, both MODIS and VIIRS instruments carry a set of on-board calibrators (OBC) for their on-orbit calibration and characterization, which include a solar diffuser (SD) and a solar diffuser stability monitor (SDSM) used primarily for the RSB calibration, a blackbody (BB) for the TEB calibration, and a SV port to collect data on a scan-by-scan basis for instrument background subtraction ${ }^{7-8}$. Not present in VIIRS, MODIS has a unique on-board spectroradiometric calibration assembly (SRCA) that can be operated in different modes to characterize sensor spectral and spatial performance. In addition to OBC observations, lunar observations collected on a regular basis have proven to be extremely important to support MODIS and VIIRS on-orbit calibration, especially for the RSB. 
Since launch, MODIS and VIIRS have also performed special calibration activities using data collected from welldesigned spacecraft maneuvers. In this paper, we focus our studies of MODIS and VIIRS on-orbit calibration and characterization using observations from spacecraft pitch maneuvers. Studies and applications using data from spacecraft yaw and roll maneuvers can be found in a number of references ${ }^{9-16}$. In the following, a brief description of MODIS and VIIRS on-orbit calibration approaches is provided, including the use of OBC, lunar observations, and data collected during spacecraft maneuvers. It is followed by specific applications to be discussed in this paper using observations from spacecraft pitch maneuvers and corresponding results. Discussion is also provided on challenges and lessons for future considerations and calibration improvements.

Table 1. VIIRS and MODIS spectral bands: spectral range and nadir spatial resolution (HSR: horizontal sampling resolution). VIIRS M1-M5, M7, and M13 are dual gain bands. Four MODIS LWIR bands (3336) are not listed in this table.

\begin{tabular}{|c|c|c|c|c|c|}
\hline VIRS Band & Spectral Range (um) & Nadir HSR (m) & MODIS Band(s) & Range & HSR \\
\hline DNB & $0.500-0.900$ & & & & \\
\hline$\bigcirc$ M1 & $0.402-0.422$ & 750 & 8 & $0.405-0.420$ & 1000 \\
\hline O MR & $0.436-0.454$ & 750 & 9 & $0.438-0.448$ & 1000 \\
\hline$O$ MB & $0.478-0.498$ & 750 & 310 & $\begin{array}{l}0.459-0.479 \\
0.483-0.493\end{array}$ & $\begin{array}{c}500 \\
1000\end{array}$ \\
\hline O M4 & $0.545-0.565$ & 750 & 4 or 12 & $\begin{array}{l}0.545-0.565 \\
0.546-0.556\end{array}$ & $\begin{array}{c}500 \\
1000\end{array}$ \\
\hline 11 & $0.600-0.680$ & 375 & 1 & $0.620-0.670$ & 250 \\
\hline$O$ MT5 & $0.662-0.682$ & 750 & 13 or 14 & $\begin{array}{l}0.662-0.672 \\
0.673-0.683\end{array}$ & $\begin{array}{l}1000 \\
1000\end{array}$ \\
\hline M6 & $0.739-0.754$ & 750 & 15 & $0.743-0.753$ & 1000 \\
\hline D & $0.846-0.885$ & 375 & 2 & $0.841-0.876$ & 250 \\
\hline$\bigcirc$ M7 & $0.846-0.885$ & 750 & 16 or 2 & $\begin{array}{l}0.862-0.877 \\
0.841-0.876\end{array}$ & $\begin{array}{c}1000 \\
250\end{array}$ \\
\hline Mi8 & $1230-1250$ & 750 & 5 & SAME & 500 \\
\hline M99 & $1.371-1.386$ & 750 & 26 & $1.360-1.390$ & 1000 \\
\hline $\mathbf{B}$ & $1.580-1.640$ & 375 & 6 & $1.628-1.652$ & 500 \\
\hline M10 & $1.580-1.640$ & 750 & 6 & $1.628-1.652$ & 500 \\
\hline M11 & $2225-2275$ & 750 & 7 & $2.105-2.155$ & 500 \\
\hline 4 & $3.550-3.930$ & 375 & 20 & $3.660-3.840$ & 1000 \\
\hline M12 & $3.660-3.840$ & 750 & 20 & SAMAE & 1000 \\
\hline M13 & $3.973-4.128$ & 750 & 21 or 22 & $\begin{array}{l}3.929-3.989 \\
3.929-3.989\end{array}$ & $\begin{array}{l}1000 \\
1000\end{array}$ \\
\hline M14 & $8.400-8.700$ & 750 & 29 & SAMA & 1000 \\
\hline M15 & $10263-11263$ & 750 & 31 & $10.780-11280$ & 1000 \\
\hline 15 & $10.500-12.400$ & 375 & 31 or 32 & $\begin{array}{l}10.780-11280 \\
11.770-12270\end{array}$ & $\begin{array}{l}1000 \\
1000\end{array}$ \\
\hline M16 & $11.538-12.488$ & 750 & 32 & $11.770-12270$ & 1000 \\
\hline
\end{tabular}

\section{MODIS AND VIIRS ON-ORBIT CALIBRATION APPROACHES}

As illustrated in Figure 2, MODIS and VIIRS on-orbit calibration and characterization approaches include the use of instrument on-board calibrators and data collected from carefully designed spacecraft maneuvers. In general, the reflectance based calibration for the RSB is tied to the on-board SD bidirectional reflectance factor (BRF), which was characterized pre-launch by the instrument vendor using reference samples traceable to the National Institute of Standards and Technology (NIST) reflectance standard. The SD on-orbit degradation is tracked by the on-board SDSM, which measures the sunlight reflected off the SD panel and the direct sunlight passing through an attenuation screen at 
nearly the same time. As expected, some of the lessons from MODIS RSB calibration have greatly benefitted the development of VIIIRS RSB calibration strategies. For MODIS and VIIRS TEB, the calibration traceability and stability are maintained using observations of the on-board BB. MODIS and VIIRS BB are of the same design with small differences, such as the number of thermistors used to measure the BB temperatures: 12 thermistors for MODIS BB compared to 6 for VIIRS BB. BB temperatures are controlled at their pre-determined setting points during nominal operations. Periodic BB warm-up and cool-down (WUCD) cycles are performed to help monitor and update, if necessary, some of the calibration parameters used for TEB on-orbit calibration. Starting from 2019, a change will be made to reduce the BB WUCD frequency from quarterly to an annual basis for both S-NPP and N-20 VIIRS.

Since launch, MODIS SRCA has been continuously operated in different modes to characterize instrument spectral and spatial performance ${ }^{17-18}$. This capability does not reside in VIIRS. As a result, alternative strategies and approaches have to be developed to overcome this deficiency. VIIRS DNB has 3 different gain stages and many aggregation modes. Its on-orbit calibration methodologies can be found in a number of references ${ }^{19}$.

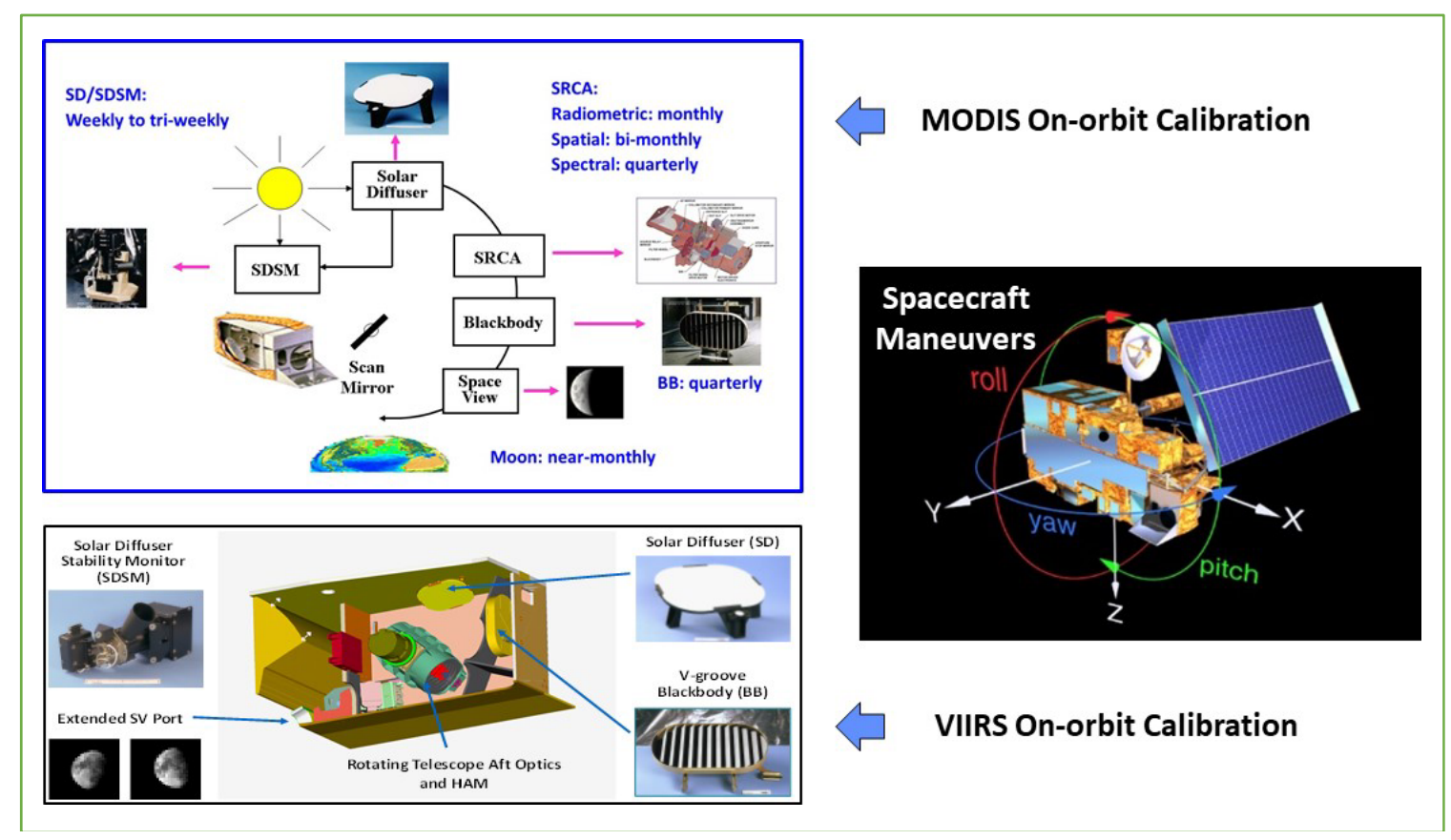

Figure 2 MODIS and VIIRS on-orbit calibration and characterization using data from on-board calibrators (left) and spacecraft maneuvers (right).

In addition to the use of instrument $\mathrm{OBC}$, lunar observations have become one of the key elements for the MODIS and VIIRS on-orbit calibration system, especially for the RSB radiometric calibration. Both MODIS and VIIRS lunar observations are performed on a regular basis through the instrument SV and via spacecraft roll maneuvers. Other types of spacecraft maneuvers, yaws and pitches, are typically performed at the mission beginning. These calibration maneuvers are carefully designed and executed to support special characterization and validation tasks that cannot be completed by the on-board calibrators during sensor nominal operation. A list of applications that can be conducted for MODIS and VIIRS using data collected during spacecraft maneuvers is provided as follows:

- SD screen transmission function (MODIS)

- SD BRF (relative, MODIS)

- SD BRF and screen transmission function (relative, VIIRS, MODIS) 
- SDSM sun view screen transmission (relative, MODIS, VIIRS)

- Radiometric stability (MODIS and VIIRS)

- Response versus scan-angle (MODIS and VIIRS TEB)

- Band-to-band registration (BBR) (MODIS, VIIRS)

- Modulation Transfer Function (MTF) (MODIS, VIIRS)

- DNB high gain dark counts (VIIRS)

MODIS and VIIRS Characterization Support Team (MCST and VCST) at NASA GSFC have developed and implemented various methodologies and algorithms to help perform the tasks listed above. This paper focuses on the use of observations made during spacecraft pitch maneuvers of MODIS and VIIRS in support of their on-orbit characterization of sensors' response versus scan-angle (RVS) for the thermal emissive bands (TEB). For Terra MODIS, it also includes the RSB RVS and BBR characterization using lunar data collected during spacecraft maneuvers.

\section{CALIBRATION AND CHARACTERIZATION USING DATA FROM PITCH MANEUVERS}

During a pitch maneuver, the spacecraft rotates around its y-axis, which is perpendicular to its velocity (x-axis) and nadir (z-axis) directions and gets an unobstructed view of the deep space, as shown in Figure 2. Since launch, Terra MODIS have performed three pitch maneuvers, during which the sensor collected data when the instrument EV port was facing the dark deep space. Two pitch maneuvers were conducted in 2003 (March 26 and April 14) and one in 2017 (August 5). Initial pitch maneuver was designed to allow Terra MODIS TEB RVS to be characterized as its pre-launch testing failed to generate valid data sets that could be used to derive a system level RVS for the TEB. The success of the first Terra pitch maneuver was followed by the second Terra pitch maneuver, which included the Moon at spacecraft nadir view. This maneuver provided an opportunity for all nadir viewing instruments on-board Terra to perform a lunar calibration at nearly the same time, thus at nearly the same lunar phase angles. The third Terra pitch maneuver, conducted after 14 years since the first one, offers an excellent opportunity to perform on-orbit verification of its TEB RVS stability. This spacecraft maneuver also included the Moon in its nadir view.

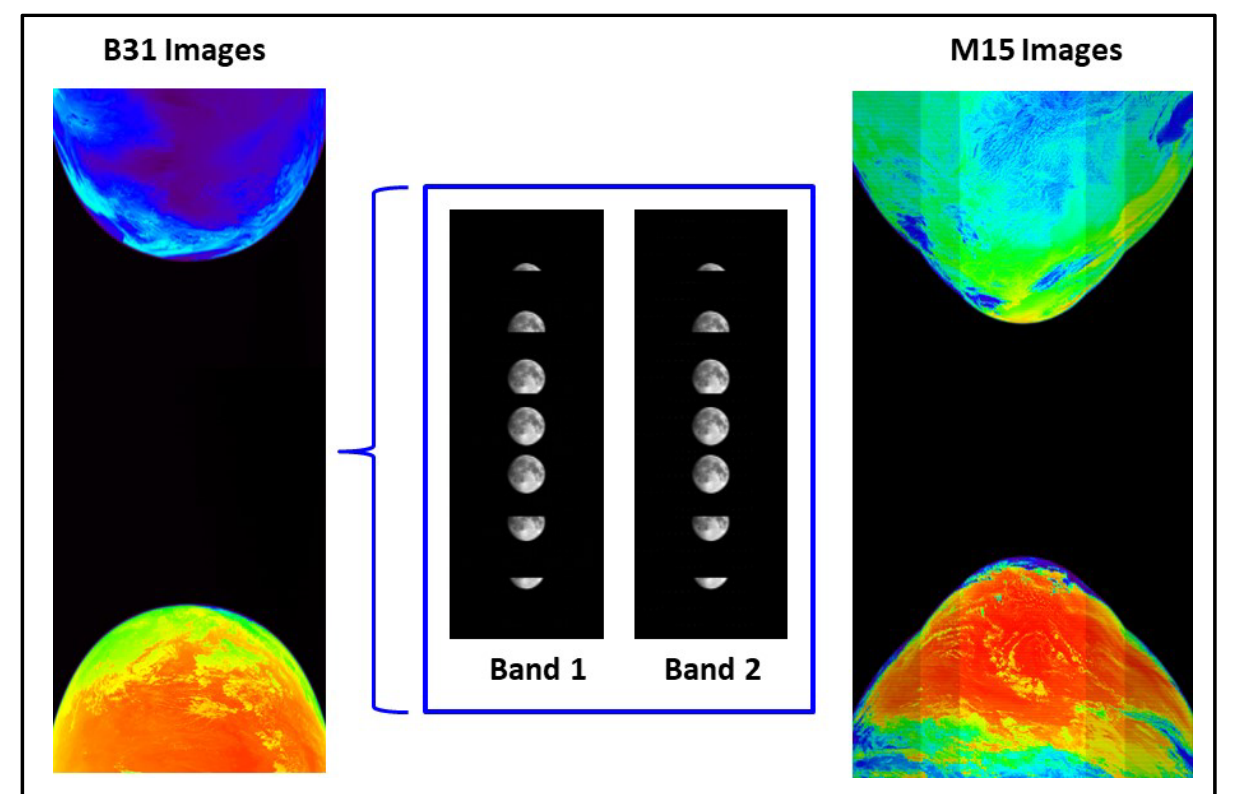

Figure 3 Image examples of Terra (band 31) and S-NPP (M15) spacecraft pitch maneuvers. 
Based on lessons learned from MODIS, S-NPP and N-20 each performed a pitch maneuver at mission beginning, February 20, 2012 for S-NPP and January 31, 2018 for N-20. The VIIRS pitch maneuvers were designed and performed in order to validate its pre-launch TEB RVS characterization. Figure 3 shows examples of the imagery obtained from both MODIS and VIIRS pitch maneuvers. The left is a false color image of MODIS band 31 taken from the pitch maneuver on August 5, 2017. The Earth limb before and after the completion of the maneuver can been seen in this image. Some of the data in between were purposely not included. Shown in the middle are lunar images of MODIS bands 1 and 2 collected during the pitch maneuver on August 5, 2017. The M15 image on the right is taken from S-NPP pitch maneuver performed on February 20, 2012. In addition to the Earth limbs, noticeable features, such as data aggregation zone and bow-tie deletion, can be easily identified.

\subsection{Thermal emissive band RVS characterization}

For the TEB, the radiance received by the detectors when looking at the deep space comes primarily from the thermal emission of the instrument components, which include the scan mirror whose thermal emission varies with the scan angle. The EV external radiance is essentially zero. Because of this, the TEB RVS can be determined by examining the relative change of the emissive radiation across the entire EV scan range, which is proportional to the change in detector's relative responses ${ }^{20-21}$. Data used in TEB RVS characterization include detector responses to the deep space and to the on-board BB.

For N-20 VIIRS, examination of all scans of its pitch maneuver data indicates that the temperatures of the two cold focal planes started to increase before there was a complete dark space view over the entire EV scan angle range. The rate of the temperature increase, however, is quite slow ( $\sim 0.026 \mathrm{~K}$ per minute) at the beginning. As a result, it is not necessary to correct the potential impact due to the slow change of the cold focal plane temperatures on the detector responses from the beginning to end of each EV scan, which takes less than 1 second. The relative RVS can be derived on a scanby-scan basis over a number of scans. Similar to the TEB RVS used in L1B calibration algorithm, the derived RVS using pitch maneuver data is fitted versus the angle of incidence (AOI) via a second order polynomial. The fitting residues (one sigma) are typically within $0.05 \%$ for all spectral bands.

\subsection{Reflective solar band RVS validation}

As previously mentioned that two of the three pitch maneuvers for Terra were scheduled such that the Moon views were available at the instrument nadir AOI, thus providing an opportunity to validate RSB on-orbit performance, particularly for the changes in the RVS by combining regularly scheduled lunar observations made at the SV AOI.

To perform this validation, we apply the RVS used in the latest MODIS L1B look-up tables (LUTs) to compute the lunar irradiance derived from pitch maneuver lunar data and that derived from regular lunar data collected a few days apart, with each normalized to the GIRO (GSICS Implementation of the ROLO) model value. The latest L1B LUTs from Collection 6.1 have included the most up-to-date RVS for the RSB. The normalization is made to account lunar irradiance difference at different phase angles. Ideally, the ratio of normalized lunar irradiance made at two AOIs should be equal to 1. Practically, the ratio, $\mathrm{R}$, is most likely close to unity, depending on the measurement uncertainty in the computed lunar irradiance, the relative RVS uncertainty between the two AOIs, and lunar model uncertainty between two different phase angles. On-orbit changes in RVS from time A to time $B$ can be evaluated using the ratio of $R_{A}$ derived at time $A$ to $R_{B}$ at time $B$.

\subsection{Band-to-band registration characterization}

The BBR is a key parameter for sensor spatial characterization. For MODIS, the BBR values in both along-scan and along-track direction have been constantly monitored using the on-board SRCA ${ }^{17}$. Similarly, one can also use the Moon, a finite point source, to characterize sensor's BBR. Several methodologies have been developed and applied by MCST to 
characterize the RSB BBR using MODIS lunar observations. The results from lunar observations have shown good agreement with those derived from SRCA measurements. These methodologies or approaches have also been adopted and improved for VIIRS BBR characterization using the Moon $^{22}$. In this study, we characterize the BBR using pitch maneuver lunar data and compare the results with that derived from regularly scheduled lunar observations made a few days apart.

\section{RESULTS AND DISCUSSION}

TEB calibration is performed using the on-board BB and, for convenience, the TEB RVS is normalize to the BB AOI (same for both MODIS and VIIRS). Figure 4 shows ratios of the pitch maneuver RVS to the pre-launch RVS (band averaged, HAM side A) for both SNPP and N20 VIIRS TEB. There is a good agreement between the pitch maneuver and pre-launch RVS, to within $0.3 \%$ for most TEB, meeting the requirement $(0.3 \%)$ specified for the RVS characterization. Similar agreement is found for the other HAM side.

By normalizing the detector dependent RVS to the band average, we can examine the detector-to-detector spreads and compare them with the pre-launch measurements. Due to the bow-tie deletion, the two edge detectors in M bands and four in I bands are excluded from on-orbit RVS characterization. Figure 5 shows an example of M15 for both S-NPP and $\mathrm{N}-20$ with detectors 3,8 , and 14 , corresponding to the beginning, middle, and end of the detector array. In general, the detector-to-detector dependent RVS differences are less than $0.1 \%$ with exception of M13 for SNPP at $0.3 \%$ for the edge detectors.
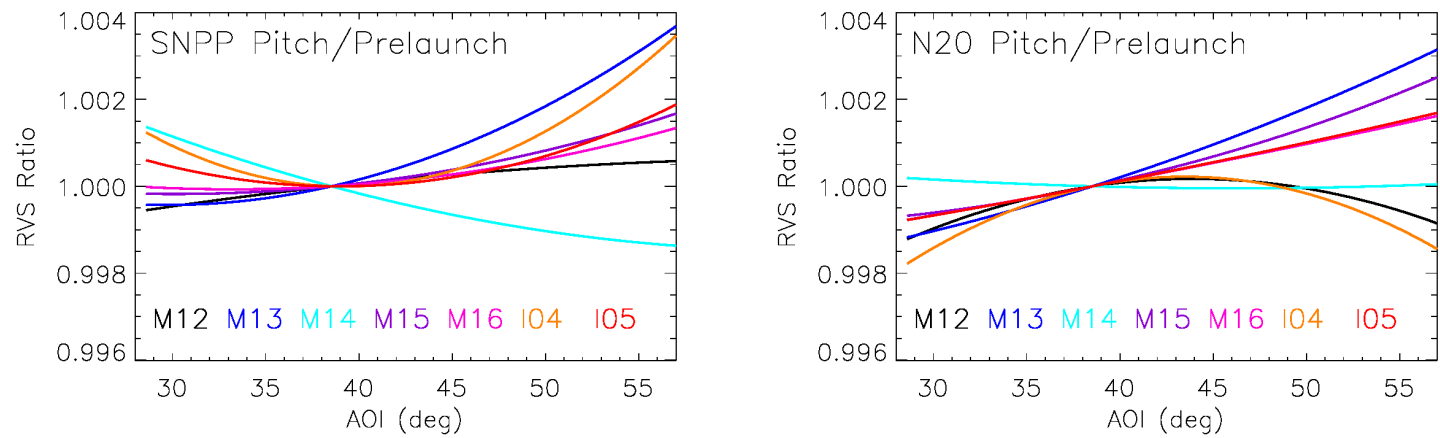

Figure 4 Ratios of pitch to pre-launch TEB RVS for SNPP (left) and NOAA-20 (right). Results are averaged for HAM side A.
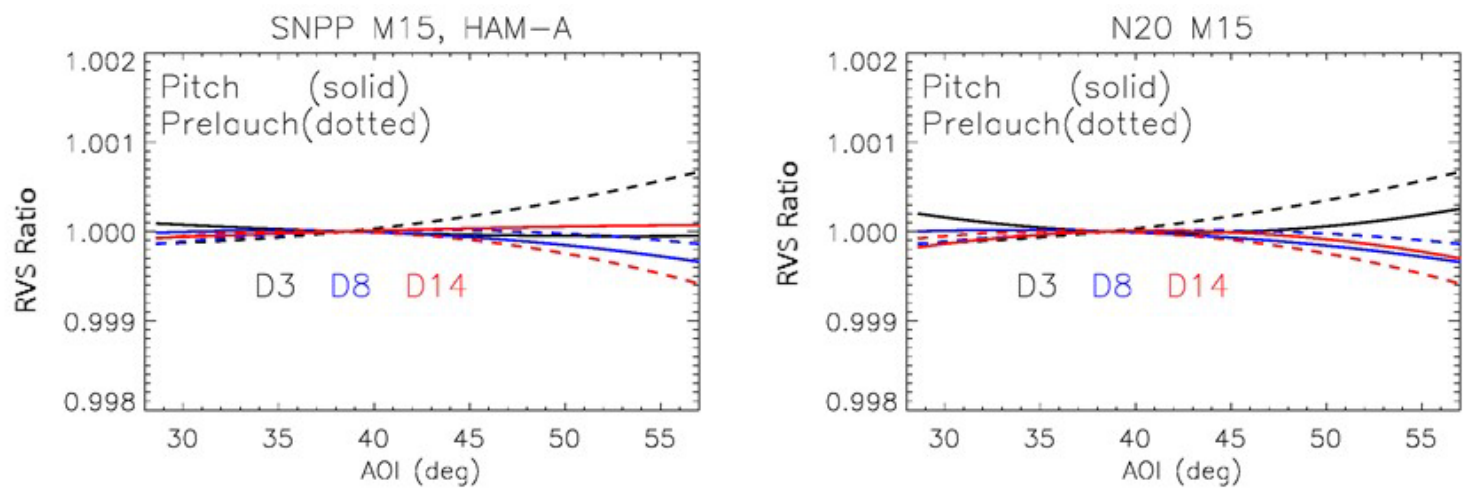

Figure 5 Comparison of M15 detector-to-detector RVS difference between results from the pitch (solid lies) and pre-launch (dotted lines) for SNPP (left) and NOAA-20 (right). Detectors 3, 8, and 14 are located at the beginning, middle and end of the detector array. 
Illustrated in Figure 6 are the ratios of Terra MODIS TEB RVS among the results (band averaged, mirror side 1) derived from all three pitch maneuvers. Terra MODIS did produce TEB RVS from pre-launch characterization. The RVS derived from the first pitch maneuver is used in Terra MODIS L1B calibration. The comparison between the first and second pitch demonstrates the repeatability of the RVS characterization as these two maneuvers were carried in less than 20 days apart during which the changes of RVS is expected to be extremely small. As show in Figure 6 (left plot) the repeatability of two RVS measurements is at the level of $0.1 \%$. As expected, the results from mirror side 2 are very similar.

The comparison between the first and third pitch shows that the differences are well within $0.2 \%$ for all bands except for band 31 at $0.3 \%$, indicating an excellent stability of Terra TEB RVS. Results from bands 27 to 30 are not included here because of the impact of electronic crosstalk. For some detectors, changes in their electronic crosstalk characteristics have been quite large.
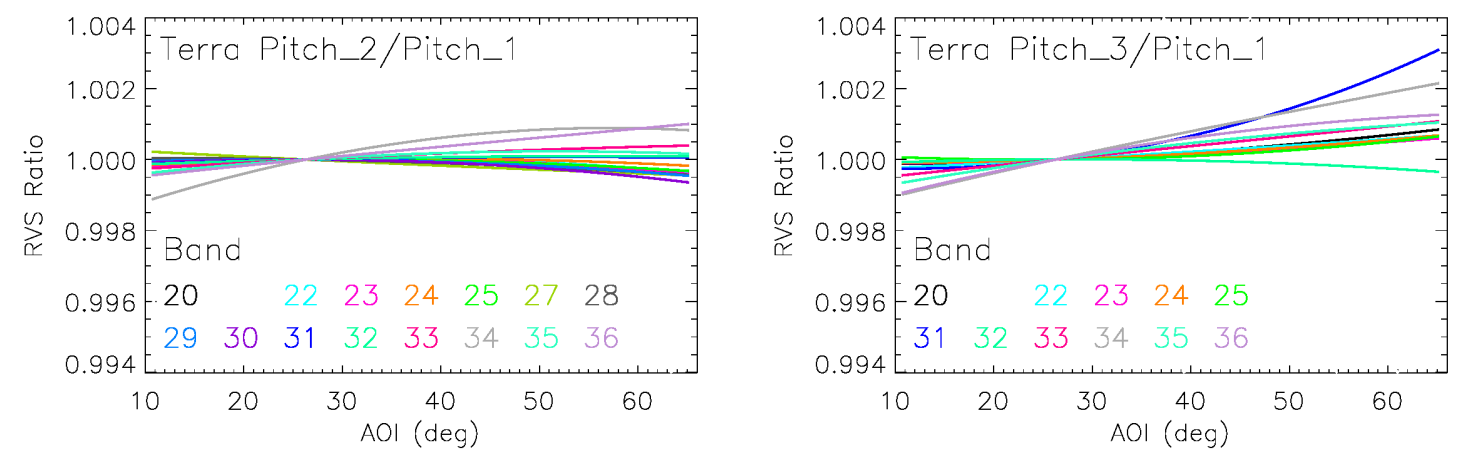

Figure 6 Ratios of RVS between the second and first pitch maneuver (left) and between the third and first pitch maneuver (right) for Terra MODIS TEB (band averaged, mirror side 1).
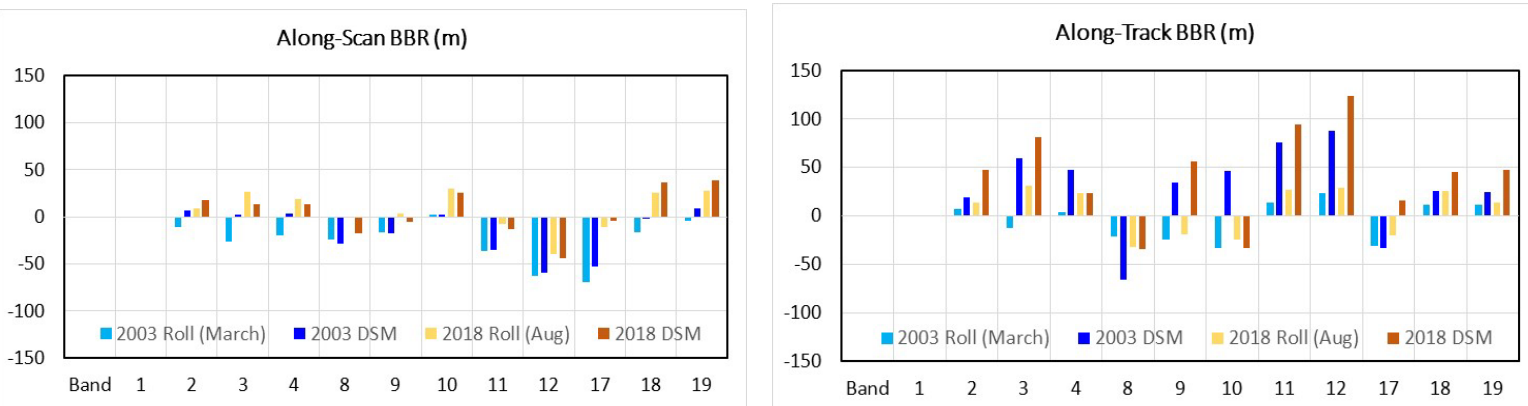

Figure 7 Along-scan (left) and along-track (right) BBR (vertical axis BBR unit: $m$ ) for the MODIS RSB from the pitch maneuver and regular lunar observations.

Figure 7 shows the BBR results (relative to band 1) for the VIS and NIR spectral bands of Terra MODIS RSB. Results derived from the deep space maneuver are plotted alongside with that derived from the scheduled lunar observations made at nearly the same time. The SWIR bands $(5,6,7$ and 26) are not included in the lunar BBR characterization as their responses are severely impacted due to the electronic crosstalk and thermal out-of-band responses. Also excluded from this analysis are the NIR bands 13-16 as their responses saturate when viewing the Moon.

Overall, a good agreement between the two lunar observations, made at different AOIs $\left(11.25^{\circ}\right.$ and $\left.38.25^{\circ}\right)$ and at different lunar phase angles $\left(\sim 55.5^{\circ}\right.$ and $\left.\sim 22^{\circ}\right)$, is observed in the along-scan direction BBR (left plot of Figure 7). The along-scan BBR values are well within the specification of $\pm 100 \mathrm{~m}$ (nadir). The right side plot of Figure 7 illustrates the BBR result in the along-track direction. Unlike the along-scan BBR results, the along-track BBR from the pitch 
maneuver lunar data shows a noticeable difference with the that obtained from scheduled lunar measurements. One of the likely causes behind the large difference is due to limited number of scans (or small oversampling factor, about 0.45 for $1 \mathrm{~km}$ bands) for the lunar observations made during pitch maneuvers. The lunar oversampling factor for the regular lunar observations made via roll maneuvers is about 4 times larger, thus more scans include the lunar views. Nevertheless, the results are as expected and well within the specified requirements.

Presented in Figure 8 are ratios of the normalized lunar irradiance from pitch maneuver lunar observation to that from regular roll maneuver lunar observation made in $2003\left(\mathrm{R}_{\mathrm{A}}\right)$ and similar ratios made in $2017\left(\mathrm{R}_{\mathrm{B}}\right)$. As described in section 4 that on-orbit changes in RSB RVS from 2003 to 2017 can be evaluated using the ratio of ratios $\left(\mathrm{R}_{\mathrm{A}} / \mathrm{R}_{\mathrm{B}}\right)$. For the shortest wavelength band $8(0.41 \mu \mathrm{m})$, the result from 2017 (in red circle) is off about $1.6 \%$ compared to the result from 2003 (blue square). Based on the current approach used to derive Terra MODIS C6.1 RVS, the apparent large difference for band 8 could be due to a number of factors, such as large changes of sensor's polarization sensitivity at short wavelengths. For the bands with wavelengths ranging from $0.44 \mu \mathrm{m}$ to $0.65 \mu \mathrm{m}$, the agreement between the 2003 and 2017 is generally within $0.5 \%$, indicating good performance of current RVS for these spectral bands. Large differences of $1.0-1.5 \%$ are also noticed for bands with wavelengths above $0.85 \mu \mathrm{m}$ and need to be carefully examined for further calibration improvements.

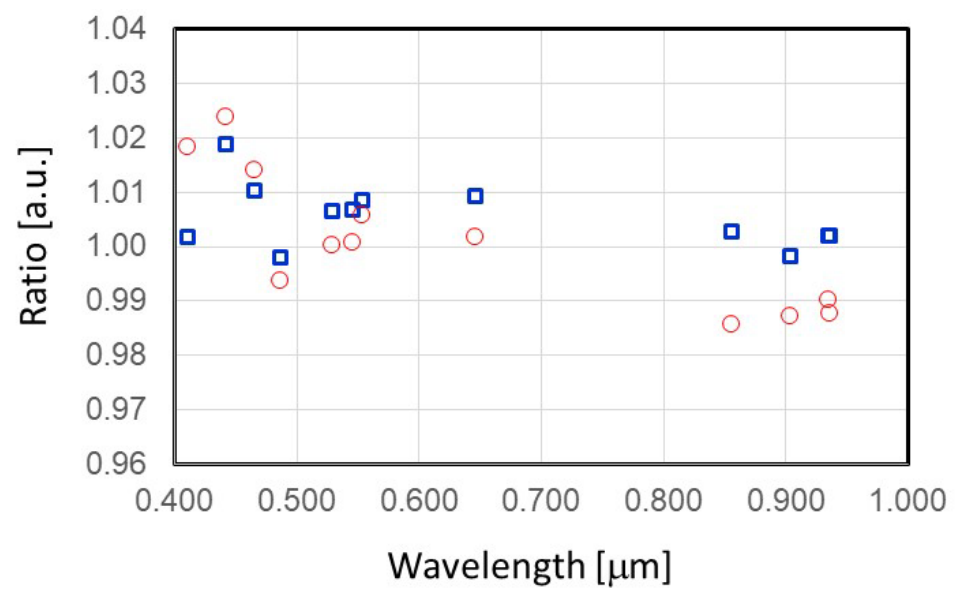

Figure 8 Ratios of the normalized lunar irradiance from pitch maneuver to roll maneuver lunar observations (2003: blue square; 2017: red circle).

As demonstrated, data collected from pitch maneuvers can be used to support sensor on-orbit calibration. For the TEB, the RVS characterization can be made very accurately at system-level on a scan-by-scan basis, covering the entire scanangle range. The lunar views included in the pitch maneuvers can also support RSB calibration and characterization. This type of pitch maneuvers has already been performed twice for Terra MODIS and needs to be considered for Aqua MODIS and future JPSS VIIRS. The pitch maneuvers performed for S-NPP and N-20 have not included the lunar view. One of concerns is loss of EV data during pitch maneuvers, especially for the TEB located on the cold focal planes, which often need longer time to return to their nominal settings following the pitch maneuvers. This is not a concern for sensors that have only RSB spectral bands.

\section{SUMMARY}

Carefully planned and executed spacecraft maneuvers can offer unique opportunities to support and enhance sensor onorbit calibration and characterization. In this paper, we have discussed several applications using data collected from Terra MODIS, S-NPP and N-20 VIIRS pitch maneuvers, including the lunar observations made during two of three Terra pitch maneuvers, and illustrated the results for TEB RVS characterization (Terra MODIS and S-NPP/N-20 VIIRS) and RSB RVS and BBR characterization (Terra NODIS). Results derived from pitch maneuvers have proven to be 
extremely valuable for validating sensor performance parameters derived (or missing) from pre-launch calibration and characterization and for monitoring changes in sensor characteristics if pitch maneuvers are performed more than once.

\section{ACKNOWLEDGEMENTS}

The authors would like to thank other members of the MODIS Characterization Support Team (MCST) and VIIRS Characterization Support Team (VCST) for their technical assistance and support.

\section{REFERENCES}

1. W.L. Barnes and V.V. Salomonson, "MODIS: A global image spectroradiometer for the Earth Observing System," Critical Reviews of Optical Science and Technology, CR47, 285-307, 1993

2. W.L. Barnes, X. Xiong, and V.V. Salomonson, "Status of Terra MODIS and Aqua MODIS," J. of Advances in Space Research, 32/11, 2099-2106, 2003

3. Salomonson V.V., W.L. Barnes, X. Xiong, S. Kempler, and E. Masuoka, "An Overview of the Earth Observing System MODIS Instrument and Associated Data Systems Performance," Proceedings of IGARSS 2002

4. Ardanuy, P., C. F. Schueler, S. W. Miller, P. S. Kealy, S. A. Cota, J. K. Haas, and C. Welsch, "NPOESS VIIRS Design Process," Proc. SPIE, vol. 4483, 22-34, 2002

5. Schueler, C. F., E. Clement, P. Ardanuy, C. Welsh, F. De Luccia, and H. Swenson, "NPOESS VIIRS sensor design overview," Proc. SPIE, vol. 4483, 11-23, 2002

6. Murphy, R.P., P. E. Ardanuy, F. Deluccia, J. E. Clement, and C. Schueler, "The visible infrared imaging radiometer suite, Earth Science Satellite Remote Sensing,” vol. 1, New York, USA: Springer-Verlag, pp. 199-223, 2006

7. X. Xiong, K. Chiang, J. Esposito, B. Guenther, and W.L. Barnes, "MODIS On-orbit Calibration and Characterization," Metrologia 40 89-92, 2003

8. Cao, C., F. Deluccia, X. Xiong, R. Wolfe, and F. Weng, "Early On-orbit Performance of the Visible Infrared Imaging Radiometer Suite (VIIRS) onboard the Suomi National Polar-orbiting Partnership (S-NPP) Satellite," IEEE Trans. Geosci. Remote Sens., vol.52, no.2, pp.1142,1156, doi: 10.1109/TGRS.2013.2247768, 2014

9. Xie, X., X. Xiong, D. Moyer, J. Sun, X. Liu, and W.L. Barnes, "Analysis of MODIS Solar Diffuser Screen Vignetting Function", Proceedings of SPIE - Earth Observing Systems X, Vol. 5882, 58820T, doi:10.1117/12.615394, 2005

10. Sun J, X. Xiong, W. Barnes, and B. Guenther, "MODIS Reflective Solar Bands On-orbit Lunar Calibration," accepted for IEEE Transactions on Geoscience and Remote Sensing, Vol. 45, No. 7, 2383-2393, 2007

11. Xiong, X., J. Sun, and W. Barnes, "Inter-comparison of On-orbit Calibration Consistency between Terra and Aqua MODIS Reflective Solar Bands Using the Moon," IEEE Geosci. Remote Sens. Let., 5(4), 778-782, 2008

12. Butler, J., X. Xiong, A. B. Barnes, F. S Patt, J. Sun, and K. Chiang, "An overview of Suomi NPP VIIRS calibration maneuvers", Proc. SPIE 8510, Earth Observing Systems XVII, 85101J, 2012; doi:10.1117/12.930993

13. McIntire, J., D. Moyer, B. Efremova, H. Oudrari, and X. Xiong, "On-Orbit Characterization of S-NPP VIIRS Transmission Functions," IEEE Transactions on Geoscience and Remote Sensing, vol. 53, issue 5, pp. 2354-2365, 2015

14. Xiong, X., J. Sun, J. Fulbright, Z. Wang, and J. Butler, "Lunar Calibration and Performance for S-NPP VIIRS Reflective Solar Bands," IEEE Transactions on Geoscience and Remote Sensing, vol. 54, issue 2, pp. 1052 - 1061, DOI:10.1109/TGRS.2015.2473665, 2016

15. X. Xiong, J. Sun, S. Xiong, and W.L. Barnes, "Using the Moon for MODIS On-orbit Spatial Characterization," Proceedings of SPIE, 5234, 480-487, 2004

16. Xiong, X., J. Sun, A. Angal, Y. Xie, T. Choi, and Z. Wang, "Results of MODIS band-to-band registration characterization using on-orbit lunar observation", Proceedings of SPIE, vol. 8153, no. 81531R, 2011

17. Xiong X, N. Che, and W.L. Barnes, "Terra MODIS On-orbit Spatial Characterization and Performance," IEEE Transactions on Geoscience and Remote Sensing, Vol. 43, No. 2, 355-365, 2005

18. Xiong X, N. Che, and W.L. Barnes, "Terra MODIS On-orbit Spectral Characterization and Performance", IEEE Transactions on Geoscience and Remote Sensing, Vol. 44, No. 8, 2198-2206, 2006 
19. Chen, H., X. Xiong, C. Sun, and K. Chiang, "Suomi-NPP VIIRS day-night band on-orbit calibration and performance", Journal of Applied Remote Sensing, vol 11(3), 360192017

20. X. Xiong, A. Wu, B. Guenther, and W.L. Barnes, "On-orbit Monitoring of MODIS Thermal Emissive Bands Response Versus Scan Angle," Proceedings of SPIE - Sensors, Systems, and Next Generation of Satellites XI, Vol. 6744, 67441I, doi: 10.1117/12.736786, 2007

21. Wu, A., X. Xiong, and K. Chiang, "Post-launch assessment of the response versus scan angle for the thermal emissive bands of visible infrared imaging radiometer suite on-board the Suomi national polar-orbiting partnership satellite", JARS, vol. 11(4), 440022017

22. Wang, Z., X. Xiong, and Y. Li, "Improved VIIRS band-to-band registration characterization based on lunar observation", Remote Sens. 2016, 8(1), 27; doi:10.3390/rs8010027 